\title{
Computer Aided Modeling and Dynamic Analysis of a New Surgical Instrument
}

\author{
Jeremy (Zheng) Li \\ School of Engineering, University of Bridgeport, Bridgeport, USA \\ Email: zhengli@bridgeport.edu
}

Received February 11, 2012; revised April 9, 2012; accepted May 8, 2012

\begin{abstract}
One major difference among surgical instruments is the level of bodily disruption and tissue trauma that surgical devices might cause the patients. This newly designed and developed surgical instrument aims at minimally invasive therapy procedure, more reliable and durable function, less operational force, and reduced manufacturing cost. The computer aided modeling and simulation have been applied to help this new instrument design and analysis. This improved new surgical instrument is designed to use in general surgery to prevent patient's vessels and tissues from being damaging due to reliable motion control of surgical clips with no unexpected clip drop. It can also be applied to surgical education purpose to educate medical students for their future surgical careers. The prototype testing indicated that the handle operational force to close surgical clips is lower than current surgical clip instruments, product manufacturing is cost-effective due to less dimensional tolerance control of this new instrument design, more reliable instrument function, and good mechanical advantage.
\end{abstract}

Keywords: Surgical Instrument; Mechanical Advantage; Suture; Occluding

\section{Introduction}

The surgical clip instruments provide function of suturing, occluding, ligating, and clamping. Proper use of surgical clip instruments can prevent surgical procedure from alignment problems [1]. The professional protocols must be strictly followed during surgical procedure and basic clinical trainings must be provided [2]. Since hardened tissues will be easily trapped between surfaces of instruments and organs, the sterilized surgical clip instruments should be kept clean to prevent tissues from hardening [3]. The new surgical instruments are currently being studied in order to minimize the trauma caused to patients, reduce the surgical recovery period, and provide surgeons with cost-effective devices [4]. Suture is a slow process and it might cause the suppuration surround cutting area and patients will have pain feeling when remove the suture [5]. This newly designed surgical clip instrument can help surgeon in their general surgical procedure to prevent surgical clip drop incident, speed up and improve the surgical process. It can also be used in surgical education to benefit future surgeons.

\section{New Surgical Clip Instrument}

In this new surgical clip instrument, the surgical clips are loaded into a protective channel in sequence. The instrument is placed at the wound edge through the open slot of the jaw pair. Driving the track channel proximally by the instrument handles can move the clips from track channel. Pushing surgical clip distally through driving channel can open jaw pair simultaneously in this new instrument. The maximum opening between the clip prongs can be achieved when instrument jaw pair is fully opened. When driving channel fully push clip distally, the surgical clip can be closed and secured onto patient's tissues or vessels.

The Figures 1 and 2 show different prototype views and Figure 3 indicates the partial view of internal mechanism of this new surgical instrument.

\section{Computational Modeling and Analysis}

The force and energy equations in this newly developed surgical clip instrument design can be determined as follows.

$$
\text { Fload } \cdot \text { Vlinear }=\mathrm{T} \cdot \omega
$$

Here, $\mathrm{T}=$ Fpivot $\cdot \mathrm{r}$,

$$
\mathrm{T} \cdot \omega=\text { Fpivot } \cdot \mathrm{r} \cdot \omega=\text { Fpivot } \cdot \text { Vangular }
$$

$$
\text { Fload } \cdot \text { Vlinear }=\text { Fpivot } \cdot \text { Vangular }
$$

Fload $=($ Vangular $/$ Vlinear $) \cdot$ Fpivot $=($ Vr $) \cdot$ Fpivot

Based on force balance in this instrument design,

$$
\text { Ffinger } \cdot \mathrm{D}=\text { Fpivot } \cdot \mathrm{R}
$$




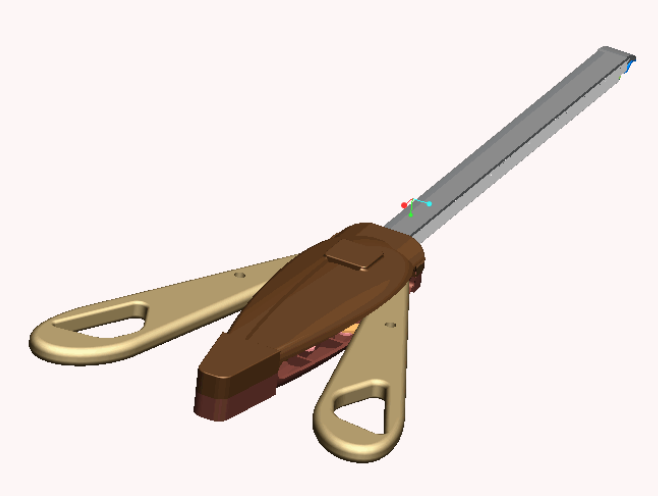

Figure 1. New surgical clip instrument rear view.

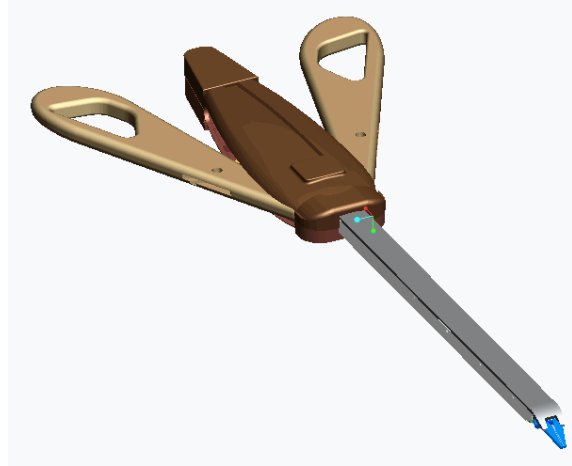

Figure 2. New surgical clip instrument front view.

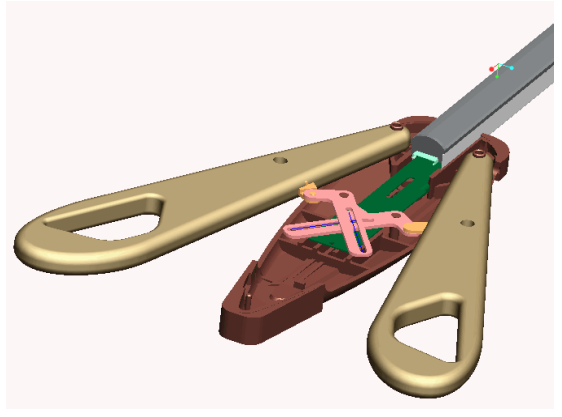

Figure 3. Internal mechanism and structure.

The load applied to surgeon's hand Ffinger can be derived from above equations. The computer aided modeling and simulation indicated the optimized instrument performance at $\mathrm{D}=3.98$ and $\mathrm{R}=1.61$.

Referring Equation (5),

$$
\begin{aligned}
& \text { Ffinger } \cdot 3.98=\text { Fpivot } \cdot 1.61 \\
& \text { Fpivot }=(3.98 / 1.61) \cdot \text { Ffinger }
\end{aligned}
$$

From Equation (4),

$$
\text { Fload }=(\mathrm{Vr}) \cdot \text { Fpivot }=(\mathrm{Vr}) \cdot(3.98 / 1.61) \cdot \text { Ffinger }
$$

The velocity ratio of $\operatorname{Vr}\left(\mathrm{V}_{\text {angular }} / \mathrm{V}_{\text {linear }}\right)$ can be determined through computer aided simulation. The results are indicated in Figure 4.
The mechanical advantage of this newly developed surgical instrument is:

$$
\begin{aligned}
\text { Mechanical advantage } & =(\mathrm{Vr}) \cdot(3.98 / 1.61) \\
& =(0.04463 / 0.03326) \cdot(3.98 / 1.61) \\
& =3.317
\end{aligned}
$$

The above result shows that surgeons only require applying $3.015 \mathrm{lbf}$ forces when $20 \mathrm{lbf}$ jaw pair force is needed to fully close the surgical clip in this new surgical instrument. This $3.015 \mathrm{lbf}$ force is lower than current specs of $4 \mathrm{lbf}$ force and it will benefit surgeons during surgical procedure.

\section{Prototype Testing}

The prototype of this new surgical instrument has been tested under different instrument design ratio shown in Table 1.

The prototype testing indicates that, under optimized design ratio of $\mathrm{D} / \mathrm{R}=3.98 / 1.61$, the mechanical advantage is 3.3194 which are very close to 3.317 from computer aided simulation. This verifies the credibility and feasibility of this new surgical instrument design.

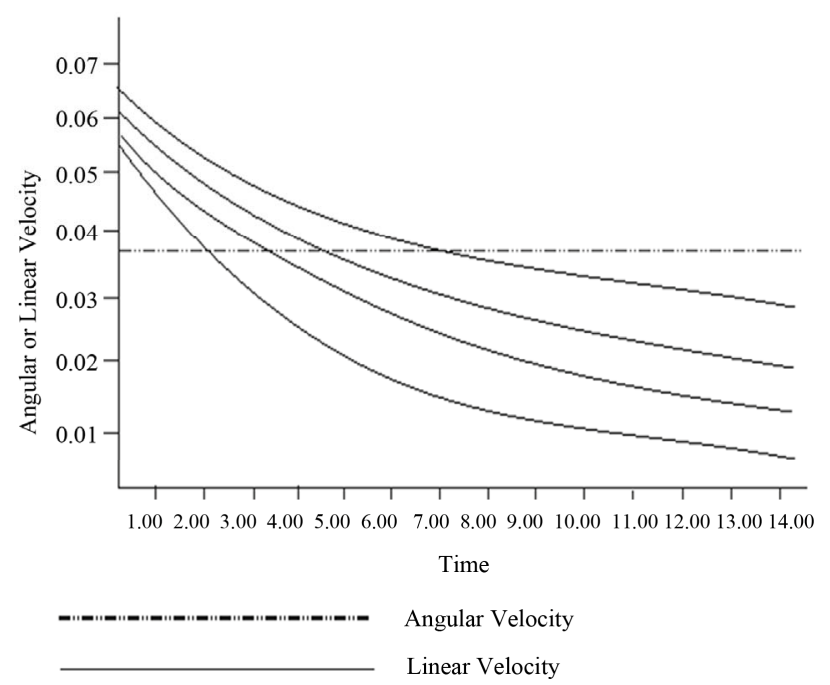

Figure 4. Clip driver linear velocity/instrument trigger angular velocity vs. operation time.

Table 1. Prototype testing under different design ratio.

\begin{tabular}{ccc}
\hline $\begin{array}{c}\mathrm{D} / \mathrm{R} \\
(\text { Design Ratio })\end{array}$ & $\begin{array}{c}\mathrm{Vr} \\
\left(\mathrm{V}_{\text {angular }} / \mathrm{V}_{\text {linear }}\right)\end{array}$ & $\begin{array}{c}\text { Mechanical } \\
\text { Advantage }\end{array}$ \\
\hline $3.86 / 1.61$ & $0.04071 / 0.03326$ & 2.9345 \\
$3.92 / 1.61$ & $0.04073 / 0.03326$ & 2.9816 \\
$3.98 / 1.61$ & $0.04466 / 0.03326$ & 3.3194 \\
$4.04 / 1.61$ & $0.03960 / 0.03326$ & 2.9876 \\
$4.10 / 1.61$ & $0.03785 / 0.03326$ & 2.8980 \\
\hline
\end{tabular}




\section{Conclusion}

This paper introduces a new surgical clip instrument design through 3D modeling, computational simulation, and prototype testing. 3D modeling and computer aided simulation can help geometrical design and dynamical analysis of new surgical instruments. The unit geometry, mechanical dynamics and visual access of this new surgical instrument have been analyzed to assist the surgeon in surgical procedures. Computer aided modeling and simulation are applied to the instrument design to determine its feasible geometry and weeping boundary. The kinematic analysis of unit mechanism can provide a systematic approach to verify instrument functionality. Both computational modeling and prototype testing verify that this new surgical instrument shows more reliable function, good mechanical advantage, cost-effective in unit production, and safe use in surgical procedure. This new surgical instrument can be applied to general surgical procedure to benefit surgeons in their surgical processes. It can also be used in surgical education to educate medical students in their future surgical careers. The prototype of this new surgical instrument will be sent to clinic fields for further evaluation and improvement.

\section{REFERENCES}

[1] S. A. Reinsberg, S. J. Doran, E. M. Charles-Edwards and
M. O. Leach, “A Complete Distortion Correction for MR Images: II. Rectification of Static-Field Inhomogeneities by Similarity-Based Profile Mapping,” Physics in Medicine and Biology, Vol. 50, No. 11, 2005, pp. 2651-2661. doi:10.1088/0031-9155/50/11/014

[2] L. Zagorchev and A. Goshtasby, “A Comparative Study of Transformation Functions for Nonrigid Image Registration," IEEE Transactions Image Processing, Vol. 15, No. 3, 2006, pp. 529-538. doi:10.1109/TIP.2005.863114

[3] M. Takao, N. Sugano, T. Nishii, H. Miki, T. Koyama, J. Masumoto, Y. Sato, S. Tamura and H. Yoshikawa, “Application of 3D-MR Image Registration to Monitoring Diseases Around the Knee Joint," Journal of Magnetic Resonance Imaging, Vol. 22, No. 5, 2005, pp. 656-660. doi:10.1002/jmri.20435

[4] F. J. S. Castro, C. Pollo, R. Meuli, P. Maeder, O. Cuisenaire, M. B. Cuadra, J.-G. Villemure and J.-P. Thiran, “A Cross Validation Study of Deep Brain Stimulation Targeting: From Experts to Atlas-Based, Segmentation-Based and Automatic Registration Algorithms,” IEEE Transactions on Medical Imaging, Vol. 25, No. 11, 2006, pp. 1440-1450. doi:10.1109/TMI.2006.882129

[5] P. Qiu, "Jump Surface Estimation, Edge Detection, and Image Restoration,” Journal of the American Statistical Association, Vol. 102, No. 478, 2007, pp. 745-756. doi:10.1198/016214507000000301 